\title{
DEPLEÇÃO DO FÓSFORO INORGÂNICO DE DIFERENTES FRAÇÕES PROVOCADA PELA EXTRAÇÃO SUCESSIVA COM RESINA EM DIFERENTES SOLOS E MANEJ OS ${ }^{(1)}$
}

\author{
D. S. RHEINHEIMER ${ }^{(2)}$, I. ANGHINONI (3) \& J . KAMINSKI (2)
}

\begin{abstract}
RESUMO
O fósforo disponível no solo é equilibrado por formas menos lábeis, as quais, a longo prazo, podem-se tornar potencialmente disponíveis às plantas. 0 trabalho teve por objetivo (a) avaliar a depleção do fósforo inorgânico de diferentes frações provocada pela extração sucessi va com resi na e (b) efetuar um balanço do fósforo no solo. Coletaram-se amostras de solo (Latossolo Vermel ho distroférrico tí́pico, Latossolo Vermel ho distrófico típico e Argissolo Vermelho distrófico típico), em três camadas $(0-2,5,2,5-7,5$ e 7,5-17,5 cm), de quatro experimentos de longa duração, nos sistemas plantio direto e cultivo convencional com diferentes sucessões de culturas. Efetuou-se o fracionamento do fósforo inorgânico pela técnica de Hedley modi ficada, antes e depois das extrações sucessivas do fósforo com resina em membrana. A fração de fósforo inorgânico extraída com $\mathrm{HCl}$ $1,0 \mathrm{~mol}^{-1}$ foi indisponível, enquanto as frações de fósforo inorgânico extraídas com $\mathrm{NaHCO}_{3}$ 0,5 mol L-1 e NaOH 0,1 mol L-1 foram lábeis, independentemente do tipo de solo, método de preparo e sucessão de cultura. Nos Latossolos, a fração de fósforo inorgânico extraída pelo $\mathrm{NaOH} 0,5 \mathrm{~mol} \mathrm{~L}^{-1}$ também foi lábil.
\end{abstract}

Termos de indexação: fósforo no solo, formas lábeis, fator quantidade.

(1) Trabal ho realizado com apoio financeiro do PRONEX-FINEP e FAPERGS. Recebido para publicação em junho de 1999 e aprovado em fevereiro de 2000.

(2) Professor do Departamento de Solos, Universidade Federal de Santa Maria - UFSM. Caixa Postal 221, CEP 97105-900 Santa Maria (RS). Bolsista do CNPq. E-mail: Danilo@creta.ccr.ufsm.br; kaminski@creta.ccr.ufsm.br

(3) Professor do Departamento de Solos, Universidade Federal do Rio Grande do Sul - UFRGS. Caixa Postal 776, CEP 91540-000 Porto Alegre (RS). Bolsista do CNPq. 


\title{
SUMMARY: DEPLETION OF INORGANIC PHOSPHORUS FRACTIONS BY SUCCESSIVE EXTRACTION WITH RESIN IN DIFFERENT SOILS AND MANAGEMENT SYSTEMS
}

\begin{abstract}
Availablesoil phosphorus is maintained by less labile form that, in thelong term, can become available to the plants. The aim of this research was to determine the depletion of vari ous inorganic phosphorus fractions after successive extraction with resin. Soil samples werecollected in four long-term experiments under notillageor conventional tillagesystems with different crop sequence. Thesoi Is (heavy clay Rhodic Hapludox, cl ay Rhodic Hapludox and clay loam Rhodic Paleudult) were coll lected from three depths, 0-2.5, 2.5-7.5 and 7.5$17.5 \mathrm{~cm}$. I norganic phosphorus was fractioned by a modified Hedl ey technique before and after successive phosphorus extraction by membrane resin. The fraction of phosphorus extracted with $1.0 \mathrm{~mol} \mathrm{~L}^{-1} \mathrm{HCl}$ is not labile. Thoseextracted with $0.5 \mathrm{~mol} \mathrm{~L}^{-1} \mathrm{NaHCO}_{3}$ and $0.1 \mathrm{~mol} \mathrm{~L}^{-1} \mathrm{NaOH}$ can be considered labile, regardless the soil type, method of cultivation and crop sequence In the Oxisols, theinorganic phosphorus fraction extracted by $0.5 \mathrm{~mol} \mathrm{~L}^{-1}$ $\mathrm{NaOH}$ is also labile
\end{abstract}

Index terms: soil phosphorus, labileforms, quantity factor.

\section{INTRODUÇÃO}

Uma abordagem moderna para a compreensão da dinâmica do fósforo é dividi-lo em frações, pelo uso de sol uções extratoras de diferentes composições e capacidades de extração, dada a complexidade do comportamento desse el emento no solo (Silva \& Raij, 1999). A primeira tentativa de fracionamento foi proposta em 1937 (Dean, 1937), pelo uso de extrações seqüenciais álcali e ácido. Mais tarde, Chang \& J ackson (1957) aprimoraram o método, introduzindo outros extratores, com vistas em separar as frações por eles identificadas como $\mathrm{Pi}-\mathrm{Al}, \mathrm{Pi}-\mathrm{Fe}, \mathrm{Pi}-\mathrm{Ca}$ e $\mathrm{Pi}-$ Fe redutor. No entanto, esse procedimento pode apresentar problemas de interpretação dos resultados, pois ocorrem readsorção durante a extração e hidrólise do fósforo orgânico. Mais recentemente, Hedley et al. (1982) propuseram uma técnica capaz de dimensionar as diferentes frações orgânicas e inorgânicas do solo de acordo com sua bi odisponibilidade, independentemente do grau de intemperismo e dos sistemas de cultivo empregados (Hedley et al., 1982; Agbenin \& Tiessen, 1994; Beck \& Sanches, 1994; Guo \& Yost, 1998). Hedley et al. (1982) eTiessen et al. (1984) consi deraram as formas extraídas por resina trocadora de ânions e bicarbonato de sódio como as formas de maior disponibilidade, ou ditas lábeis. J á aquel as extraídas com NaOH 0,1 mol L-1 comporiam a fração moderadamentelábil. As frações de fósforo extraídas por ultra-som, $\mathrm{NaOH} 0,5 \mathrm{~mol} \mathrm{L-1,} \mathrm{HCl} 1,0$ mol L-1 e o residual, seriam consideradas não-lábeis. Isto estende-se às frações de fósforo orgânico detectadas pel os mesmos extratores.

A publicação do método de Hedley et al. (1982) é um divisor metodológico importante, pois, a partir dele, outros trabal hos têm sido realizados com sol os de regi ões temperadas e, mais recentemente, al guns com sol os bem intemperizados (Cross \& Schlesinger, 1995). O interessena aplicação desse método para o estudo da dinâmica do fósforo em solos das regiões tropicais e subtropicais tem aumentado e envolve, especial mente, a adição de fertilizantes (Ball-Coel ho et al., 1993; Beck \& Sanchez, 1994; Schmidt et al., 1996; Zhang \& MacKenzie, 1997; Araújo \& Salcedo, 1997; Guo \& Yost, 1998) e o manejo do solo (Selles et al., 1997). Estes trabalhos demonstram que as formas mais disponíveis são tamponadas, principalmente pel o fósforo inorgânico extraído pelo $\mathrm{NaOH}$ 0,1 mol L-1, o qual compõe, basicamente, o fator quantidade. A magnitude dessa fração aumenta quando as adições superam as saídas e diminui em solos cultivados com baixa reposição do fósforo exportado. $\mathrm{O}$ acúmulo, ou o dedínio, do fósforo do solo depende do balanço entre as adições e as retiradas do sistema. O fator quantidade é monitorado pel os métodos convencionais de análise de fósforo, mas para os solos bem intemperizados há uma subestimação do seu valor, pois extrações sucessivas com o mesmo extrator continuam retirando fósforo do solo (Campello et al., 1994; McKean \& Warren, 1996). Assim, ofator quanti dade poderia ser mais bem monitoradotanto pela extração com NaOH 0,1 mol L-1 (Ball-Coel ho et al., 1993; Beck \& Sanchez, 1994; Schmidt et al., 1996; Araújo \& Salcedo, 1997; Zhang \& MacKenzie, 1997; Guo \& Yost, 1998) como pela extração sucessiva com resina trocadora de ânions (Haggar et al., 1991; Campello et al., 1994; McK ean \& Warren, 1996).

Este trabalho teve por objetivo (a) avaliar a depleção nos teores de fósforo inorgânico das frações obtidas com a técnica de Hedley após extrações sucessivas com resina trocadora de ânion em membrana e (b) efetuar um bal anço do fósforo no solo, 
usando amostras de solos de quatro experimentos delonga duração, envolvendo diferentes métodos de preparo e sucessões de culturas.

\section{MATE RIAL E MÉTODOS}

As amostras de solo utilizadas neste trabalho foram coletadas de quatro experimentos de longa duração, instalados sobre três tipos de solos, com objetivo de estudar os efeitos de métodos de preparo do sol o e de sistemas de culturas na recuperação da qualidade do solo.

O primeiro experimento foi instalado, em 1979, num Latossolo Vermelho distroférrico típico muito argil oso substrato basalto (LVdf - Rhodic Hapludox) no Centro de Atividades Agrícolas e Florestais da Cooperativa Tritícola de Santo Ângelo (RS). A área tinha sido anteriormente cultivada por 15 anos em cultivo convencional eapresentava avançado estado de degradaçãofísica. Na instalação do experimento, em 1985, e, posteriormente, em 1992, aplicou-se cal cário na dose para elevar o pH até 6,0 (método SMP). A adubação usada a partir da instalação do experimento seguiu a recomendação técnica específica a cada cultura. As amostras de sol o foram coletadas nas parcelas do sistema plantio direto (SPD) e nas do cultivo convencional (SCC), com as sucessões de culturas aveia (Avena strigosa Screb)/ milho (Zea mays L.) e trigo (Triticum aestivum L.)/ soja (Glycinemax (L.) Merrill). No SPD, col etaramse amostras, também, das parcelas com a rotação envolvendo as culturas: trigo, soja, tremoço (Lupinus angustifolium L.), milho, sorgo (Sorghum bicolor L.) e aveia preta +trevo (Trifolium repens L.). As amostras de solo foram col etadas nas parcelas que nunca receberam adubação nitrogenada, na expectativa de que maior quantidade de fósforo seria acumulada no solo, uma vez que a exportação pelas colheitas era menor. Maiores detalhes podem ser obtidos em Dalla Rosa (1981), Merten (1988) eDalla Rosa et al. (1991).

O segundo experimento foi instalado, em 1983, num Latossolo Vermelho distrófico típico argiloso substrato basalto (LVd - Rhodic Hapludox) no Centro Nacional dePesquisa doTrigo-EMBRAPA, em Passo Fundo (RS). As amostras foram coletadas nos tratamentos SPD eSCC enos blocos cultivados com sorgo e soja, de uma seqüência de culturas alternadas no tempo, envolvendo ervilhaca (Vicia sativa L.), milho, sorgo, aveia preta, soja e cevada (Hordeum vulgare L.). O calcário foi aplicado na instalação do experimento e reaplicado, em 1989, com incorporação ao solo, na dose recomendada para elevação o pH até 6,0 e a adubação usada seguiu as recomendações técnicas. Maiores detal hes podem ser obtidos em Selles et al. (1997) e Denardin \& Kochhann (1998).
O terceiro experimento foi instalado, em 1983, num Argissol o Vermel ho distrófico franco-argil osoarenoso (PVd - Rhodic Paleudult) degradado fisicamente, em Eldorado do Sul (RS), pelo Departamento de Solos da Universidade Federal do Rio Grande do Sul. Amostrou-se o solo das parcelas sem nitrogênio e solo descompactado, nas sucessões de culturas aveia/ milho, aveia + ervilhaca/milho + caupi (Vigna unguiculata subs unguiculata (L.) Walp) e guandu (Cajanus cajan (L.) Millps) + milho, sob SPD.

O quarto experimento foi instalado, em 1985, em área adjacente ao experimento descrito anteriormente. $\mathrm{O}$ sol o foi amostrado nos tratamentos SPD e SCC nas sucessões de culturas aveia/mil ho e aveia + ervilhaca/milho + caupi, sem aplicação de nitrogênio. Maiores detal hes podem ser obtidos em Testa (1989), Bayer (1992) e Amado et al. (1999).

Em maio de 1997, logo após o preparo, col etaramseamostras desolo nos quatro experimentos, em duas repetições de cada tratamento, nas profundidades de0-2,5, 2,5-7,5e 7,5-17,5 cm. Cada amostra foi composta pela homogeneização de duas subamostras oriundas de trincheiras de $50 \times 10 \times 17,5 \mathrm{~cm}$, coletadas perpendicularmenteàs linhas de semeadura. $\mathrm{O}$ solo foi seco ao ar e peneirado em malha de $1 \mathrm{~mm}$.

Para ofracionamento do fósforo inorgânico, usouse o método de Hedley et al. (1982) (resina, $\mathrm{NaHCO}_{3}$ $0,5 \mathrm{~mol} \mathrm{~L}-1, \mathrm{NaOH} 0,1 \mathrm{~mol} \mathrm{~L}^{-1}, \mathrm{HCl} 1,0 \mathrm{~mol}^{-1} \mathrm{e}$ $\mathrm{H}_{2} \mathrm{SO}_{4}+\mathrm{H}_{2} \mathrm{O}_{2}$ ), com inclusão de uma extração inicial com resina trocadora de ânion em membrana (AR 103 QDP 434 - Ionics, Inc.) e uma segunda extração com $\mathrm{NaOH}$ 0,5 mol L-1, após o ácido clorídrico (Condron et al., 1985). O fósforo inorgânico dos extratos foi determinado, usando-se o procedimento descrito por Dick \& Tabatabai (1977).

O fósforo potencialmente lábil foi estimado pela extração sucessiva com resina trocadora de ânion em membrana (McKean \& Warren, 1996). Este procedimento consistiu em pesar $0,500 \mathrm{~g}$ de solo, colocando-o em tubo de centrífuga com tampa com rosca. Adicionaram-se $30 \mathrm{~mL}$ de água destilada e uma membrana $(1,5 \times 5,0 \mathrm{~cm})$ de resina saturada com $\mathrm{NaHCO}_{3}$ 0,5 mol L-1. Agitou-se o material por 16 horas no agitador sem fim. No final deste período, as membranas de resina foram lavadas com jato de água destilada ecol ocadas em frascos "snap-cap" com $50 \mathrm{~mL}$ de $\mathrm{HCl}$ 0,5 mol L-1. As membranas de resina permaneceram nesta solução por 90 min e depois foram agitadas por $30 \mathrm{~min}$ (horizontal, 100120 oscilações/min). O teor de fósforo contido no extrato foi determinado de acordo com Murphy \& Riley (1962). Repetiu-se a extração por onze vezes, para que o teor de fósforo extraído fosse próximo de zero. O sol o permanecia em repouso, saturado com água, por um período de 4 a 17 dias entre cada extração. No resíduo, procedeu-seao fracionamento do fósforo inorgânico, usando os mesmos extratores adicionados nas amostras não submeti das à extração sucessiva com resina. 
Realizou-se um balanço do fósforo para o Latossolo Vermelho distroférrico típico. O fósforo total adicionado foi calculado com base na dose aplicada em cada cultivo e o número de cultivos; o fósforo exportado foi calculado com base na produtividade média de cada cultura obtida nos referidos tratamentos, no número de cultivos e no teor médio de fósforo nos grãos. Para estimar o fósforo perdido por erosão, considerou-se o teor de fósforo total do solo em cada tratamento e pressupôsse uma perda média de sol o de 1 e 3 t ha-1 ano-1, para oSPD eSCC, e uma taxa de enriquecimento de 2 e $1,5 \mathrm{mg} \mathrm{dm}^{-3}$ de $\mathrm{P}$ no sedimento por $\mathrm{mg} \mathrm{dm}^{-3}$ de $\mathrm{P}$ no solo, para os mesmos sistemas (Senganfredo, 1995). O fósforo potencialmente disponível foi obtido pelo somatório das extrações sucessivas com resina em membrana. A diferença entre o fósforo adicionado e aquele exportado pelos grãos, perdido por erosão e que permaneceu no sol o potencialmente disponível, foi considerada como fósforo recuperado.

Os valores de fósforo extraído pelo $\mathrm{NaHCO}_{3}$ $0,5 \mathrm{~mol} \mathrm{~L}^{-1}, \mathrm{NaOH} 0,1$ e 0,5 mol L-1 e HCl 1,0 mol L-1 foram submeti dos à análise de variância, para cada solo separadamente, considerando os métodos de preparo, as sucessões de culturas, as camadas amostradas e os teores obtidos antes e depois das extrações sucessivas com a resina em membrana. As médias, antes e depois das extrações sucessivas com a resina em membrana, dos tratamentos dos métodos de preparo, sucessões deculturas e camadas amostradas foram comparadas pelo teste de Tukey $(p<0,05)$. Nos três primeiros experimentos, consideraram-se, como parcelas principais, os métodos de preparo do solo e, como subparcelas, os sistemas de culturas. No quarto experimento, só foram considerados os sistemas de culturas. As camadas amostradas foram consideradas como tratamentos, com restrição à al eatorização.

\section{RESULTADOS E DISCUSSÃO}

O teor de fósforo inorgânico extraído pelo $\mathrm{NaHCO}_{3}$ diminuiu $(p<0,05)$ nos quatro experimentos após as extrações sucessivas com a resina em membrana, comparativamente ao seu val or inicial. Cabe salientar que os valores antes considerados (Quadro 1) referem-se, na verdade, aos obtidos após

Quadro 1. Fósforo extraído pelo $\mathrm{NaHCO}_{3} 0,5 \mathrm{~mol} \mathrm{~L}^{-1}$ antes e depois das extrações sucessivas com resina trocadora de ânion em membrana, considerando diferentes tipos de solo, métodos de preparo, sucessões de culturas e camadas amostradas

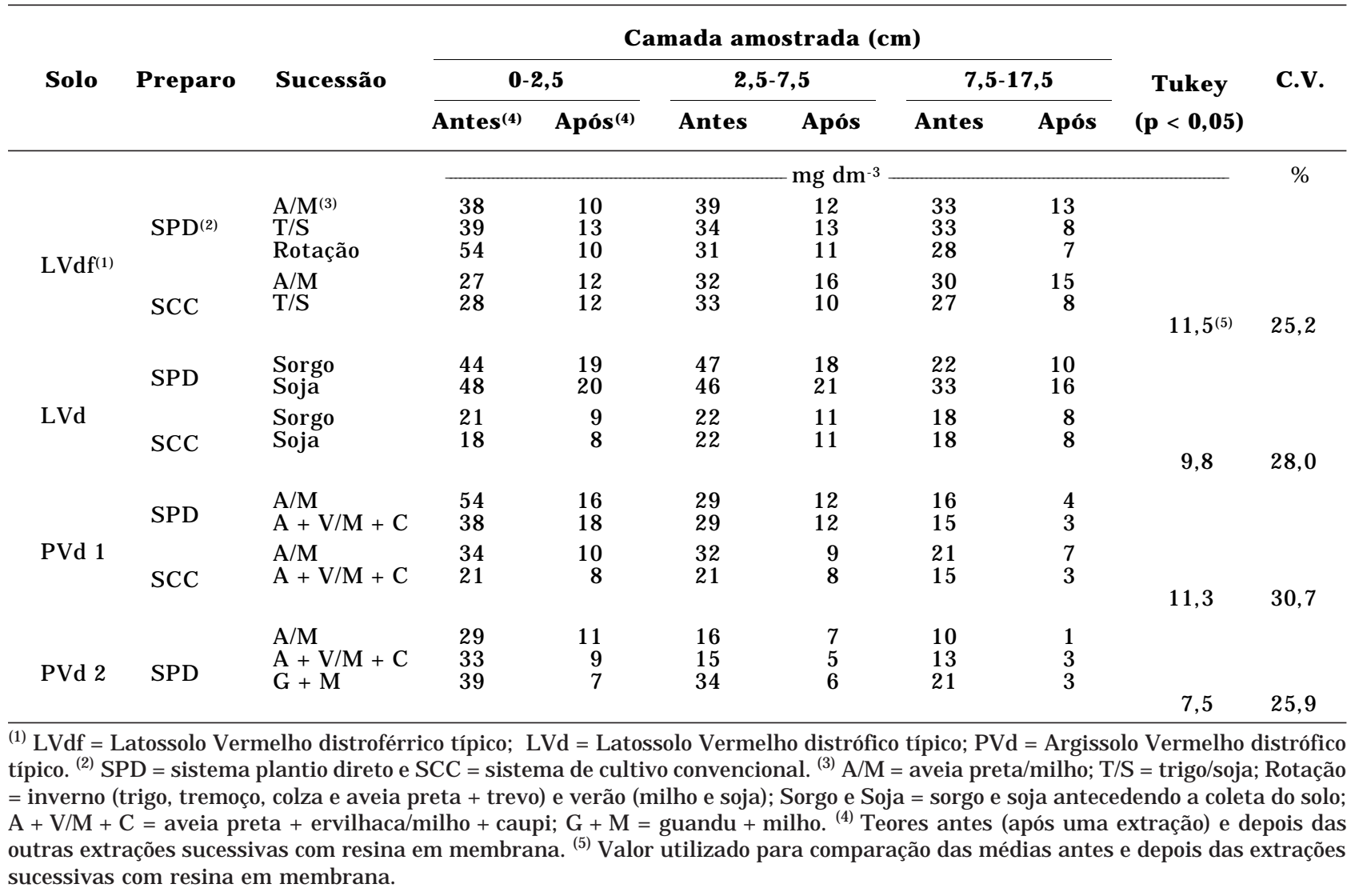


uma extração com resina e, como aproximadamente $50 \%$ do total é retirado na primeira extração, podese inferir que os teores, sem extração prévia com a resina, do $\mathrm{Pi}-\mathrm{NaHCO}_{3}$ seriam mais el evados. Assim mesmo, os teores, médias dos métodos de preparo de solo, sucessões de culturas e camadas amostradas diminuíram de 34 para 11, de 30 para 13, de 27 para 9 e de 23 para $6 \mathrm{mg} \mathrm{dm}^{-3}$, para o 1ㅇ, 20, 3으 e 4은 experimentos, respectivamente (Quadro 1), confirmandoa labilidade dessa fração, a qual contribui para o tamponamento do fósforo potencialmente disponível à biomassa, independentemente do tipo de solo, método de preparo, sucessão de cultura e camada amostrada.

Por outrolado, oteor de fósforo extraído pelo $\mathrm{HCl}$ 1,0 mol L-1, usado em seqüência à resina, $\mathrm{NaHCO}_{3}$ $0,5 \mathrm{~mol} \mathrm{~L}^{-1}$ e $\mathrm{NaOH} 0,1 \mathrm{~mol} \mathrm{L-1}$, não se modificou $(p<0,05)$ com as extrações sucessivas com resina em membrana (Quadro 2). Essa fração representa média dos métodos de preparo de sol o, sucessões de culturas e camadas amostradas, 32, 23, 19 e $22 \mathrm{mg} \mathrm{dm}^{-3}$, para o 10, 2ㅇ, 3o e 40 experimentos, respectivamente (Quadro 2 ) e é considerada não lábil, estável ou refratária (Hedley et al., 1982;
Tiessen et al., 1984). Possivel mente, écomposta por fosfatos de cálcio neoformados a partir da precipitação na solução do solo, decorrente da alta concentração de fósforo e cálcio (Magid, 1993). O fósforo contido nessa fração só se tornará disponível no sol o se ocorrer um abaixamento do pH e, ou, dos teores de fósforo e de cál cio em solução, o que pode ser observado na região da rizosfera (Smiley, 1974).

O teor de fósforo extraído pelo $\mathrm{NaOH} 0,1 \mathrm{~mol} \mathrm{L-1}$ diminuiu substancialmente após as extrações sucessivas com resina em membrana, sendo significativo $(p<0,05)$ para a maioria dos tratamentos (Quadro 3). Em média, os teores diminuíram de 139 para 99, de 273 para 156, de 158 para 97 e de 160 para $115 \mathrm{mg} \mathrm{dm}^{-3}$, para o 1ㅇ, 2, 3ㅇe 40 experimentos, respectivamente. Portanto, essa fração de fósforo inorgânico é principal tamponante do fósforo potencialmente disponível. NoLVdf e LVd, cujos teores de argila (680 e $530 \mathrm{~g} \mathrm{~kg}^{-1}$ ) e óxidos de ferro extraído por ditionito-citrato-bicarbonato (246 e56 g kg-1) sãomuito maiores comparativamenteaos do Argissolo ( $220 \mathrm{~g} \mathrm{~kg}^{-1}$ de argila e $36 \mathrm{~g} \mathrm{~kg}^{-1} \mathrm{de} \mathrm{Fe}_{\mathrm{d}}$ ), a quantidade de fósforo extraída com o $\mathrm{NaOH}$ 0,5 mol L-1, também, diminuiu de 149 para 97 e de

Quadro 2. Fósforo extraído pelo $\mathrm{HCl} 1,0 \mathrm{~mol} \mathrm{~L}^{-1}$ antes e depois das extrações sucessivas com resina trocadora de ânion em membrana, considerando diferentes tipos de solo, métodos de preparo, sucessões de culturas e camadas amostradas

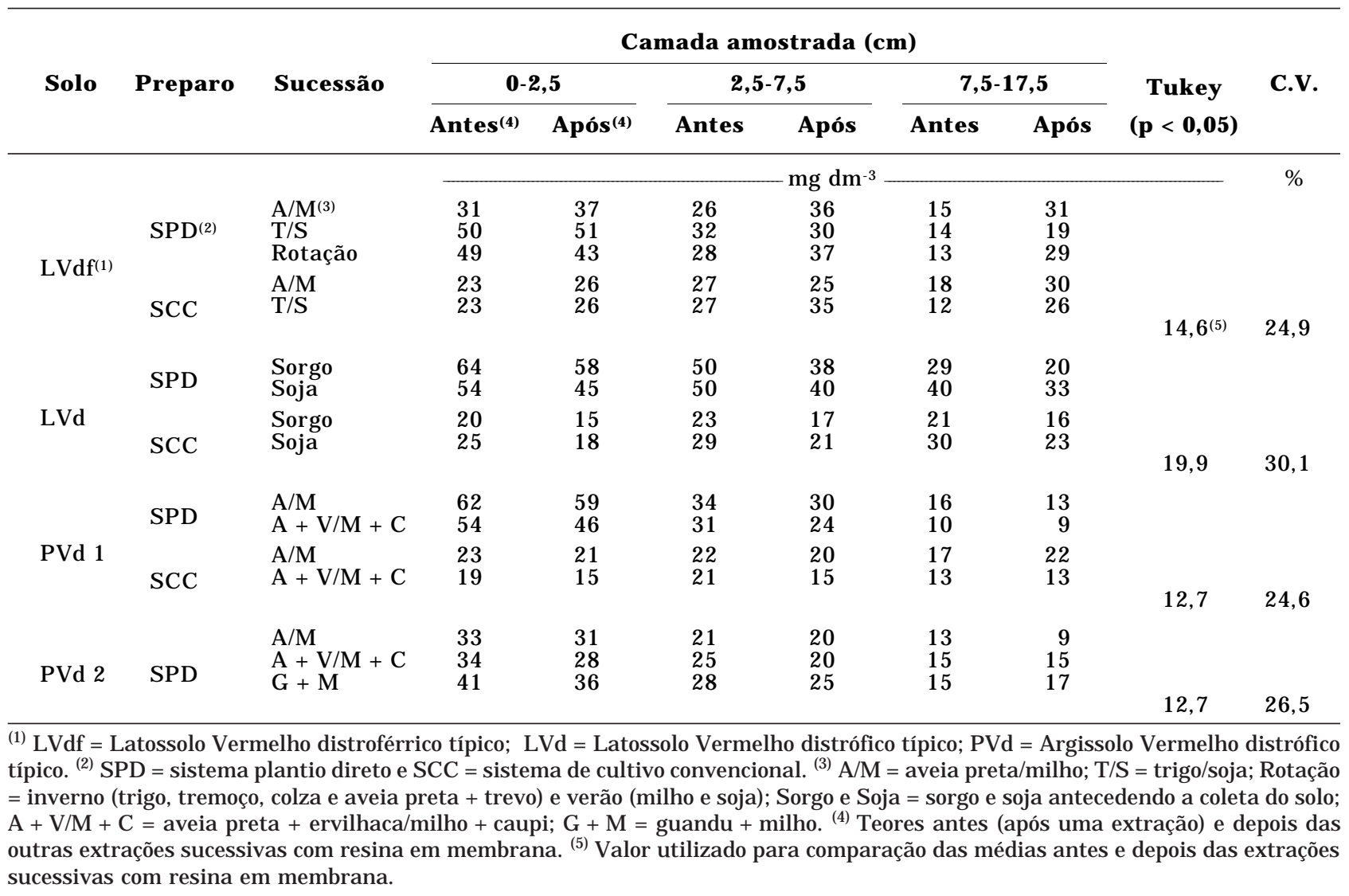


Quadro 3. Fósforo extraído pelo $\mathrm{NaOH} 0,1 \mathrm{~mol} \mathrm{~L}^{-1}$ antes e depois das extrações sucessivas com resina trocadora de ânion em membrana, considerando diferentes tipos de solo, métodos de preparo, sucessões de culturas e camadas amostradas

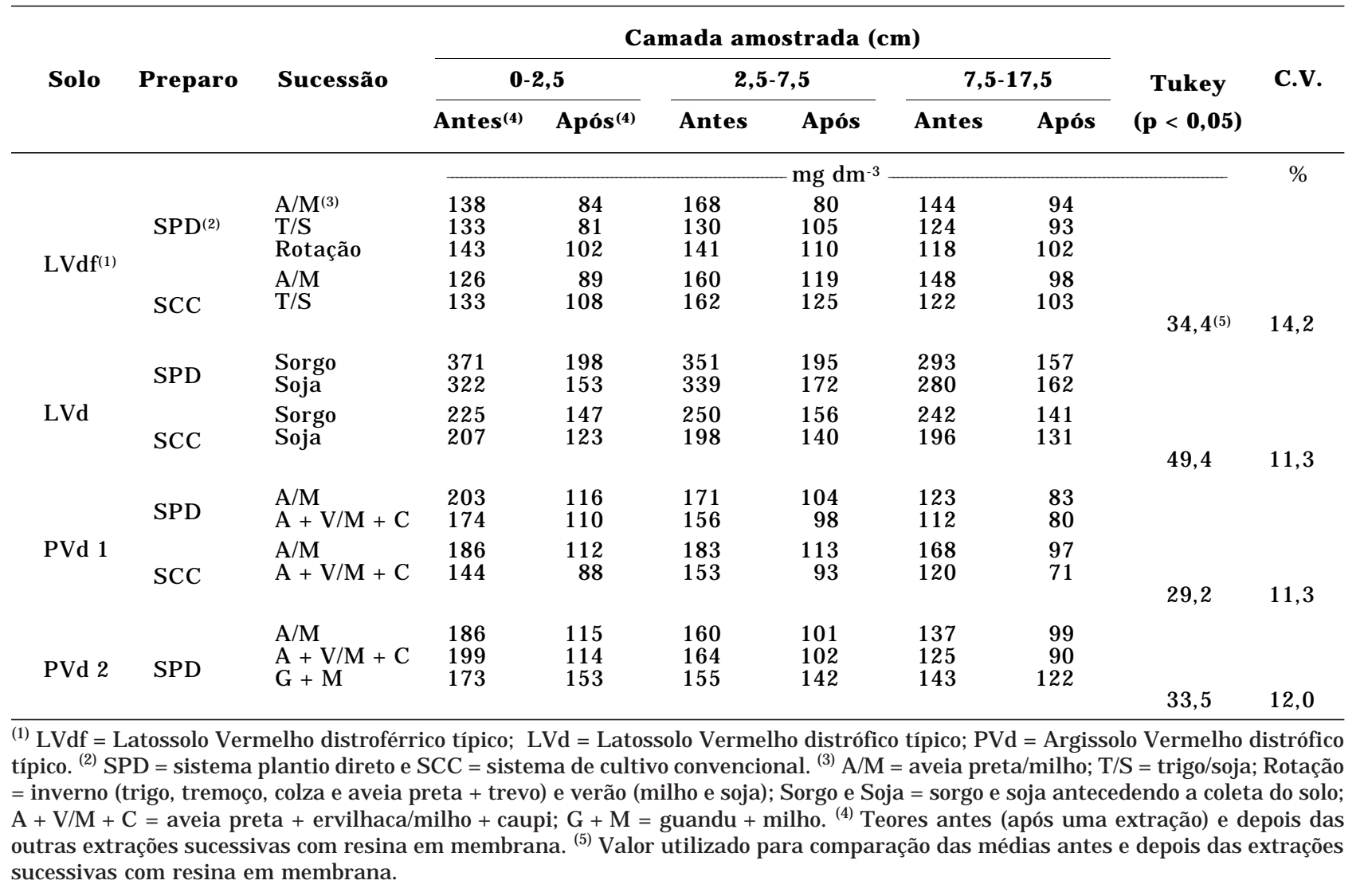

109 para $90 \mathrm{mg} \mathrm{dm}^{-3}$, para o LVdf e LVd, respectivamente (Quadro 4), representando um potencial de disponibilidade. Isso mostra que, em qualquer tipo de solo ou manejo empregado, as frações extraídas com $\mathrm{NaOH}$ foram as que mais contribuíram notamponamento do fósforo disponível, como também fora observado em outros trabalhos (Ball-Coelho et al., 1993; Beck \& Sanchez, 1994; Schmidt et al., 1996; Zhang \& MacKenzie, 1997; Araújo \& Salcedo, 1997; Guo \& Yost, 1998; Selles et al., 1997).

Desse modo, o fósforo inorgânico extraído pelo $\mathrm{NaOH}(0,1$ e 0,5 mol L-1) foi o principal tamponante do fator quantidade, estimado pelas extrações sucessivas com a resina, representando, em média, $85,85,76$ e $69 \%$, para o 1ㅇ, 2ㅇ, 3ㅇ e 4ㅇe experimentos, respectivamente. O Pi-NaHCO 3 contribuiu com 20, 10,22 e $26 \%$ para os referidos experimentos, enquanto o $\mathrm{Pi}-\mathrm{HCl}$ não é importante para o fator quantidade (Quadro 5).

Os métodos de análise para fósforo disponível do sol o em uso no Brasil extraem uma pequena porção do "pool" do fósforo total do solo. Esses apresentam moderada correlação com a quantidade de fósforo absorvida pela planta (Kroth, 1998; Silva \& Raij, 1999), pois as taxas de dessorção são, na maioria dos casos, desconsideradas no planejamento das adubações. A capacidade preditiva dos métodos de análise poderia ser melhorada, combinando-se os resultados obtidos pelo método usual à estimativa do fósforo potencialmente disponível, ou àquelas formas que o tamponam. No caso de solos com el evado grau de intemperização e que tenham recebido adubação durante vários ciclos de cultivo, poder-se-ia medir a fração de fósforo inorgânico potencialmente biodisponível, solúvel em $\mathrm{NaOH}$, o que representaria mais adequadamente o fator quantidade. Tal procedimento poderia melhorar as práticas de adubação fosfatada nas diferentes situações de manejo, tipos de solo e espécies cultivadas (Sanyal \& Datta, 1991; Guo \& Yost, 1998).

Os solos dos experimentos utilizados neste trabal ho foram anteriormente cultivados por vários anos de modo convencional e receberam adubações fosfatadas em todos os cultivos durante sua condução. A partir dos dados experimentais obtidos no sol o LV df e de al gumas pressuposições, como as mencionadas no Material e Métodos, elaborou-se uma estimativa do balanço de fósforo (Quadro 6). 
Quadro 4. Fósforo extraído pelo $\mathrm{NaOH} 0,5 \mathrm{~mol} \mathrm{~L}^{-1}$ antes e depois das extrações sucessivas com resina trocadora de ânion em membrana, considerando diferentes tipos de solo, métodos de preparo, sucessões de culturas e camadas amostradas

\begin{tabular}{|c|c|c|c|c|c|c|c|c|c|c|}
\hline \multirow{3}{*}{ Solo } & \multirow{3}{*}{ Preparo } & \multirow{3}{*}{ Sucessão } & \multicolumn{6}{|c|}{ Camada amostrada $(\mathrm{cm})$} & \multirow{3}{*}{$\begin{array}{c}\text { Tukey } \\
(p<0,05)\end{array}$} & \multirow{3}{*}{ C.V. } \\
\hline & & & \multicolumn{2}{|c|}{$0-2,5$} & \multicolumn{2}{|c|}{$2,5-7,5$} & \multicolumn{2}{|c|}{$7,5-17,5$} & & \\
\hline & & & Antes(4) & Após(4) & Antes & Após & Antes & Após & & \\
\hline \multirow{5}{*}{$\operatorname{LVdf}(1)$} & & & \multicolumn{6}{|c|}{$-\mathrm{mg} \mathrm{dm}^{-3}$} & \multirow{5}{*}{$22,7^{(5)}$} & \multirow[t]{2}{*}{$\%$} \\
\hline & $\mathrm{SPD}^{(2)}$ & $\begin{array}{l}\text { A/M(3) } \\
\text { T/S } \\
\text { Rotação }\end{array}$ & $\begin{array}{l}150 \\
172 \\
171\end{array}$ & $\begin{array}{l}110 \\
121 \\
104\end{array}$ & $\begin{array}{l}152 \\
164 \\
160\end{array}$ & $\begin{array}{r}102 \\
93 \\
105\end{array}$ & $\begin{array}{l}134 \\
126 \\
119\end{array}$ & $\begin{array}{l}94 \\
83 \\
88\end{array}$ & & \\
\hline & & $A / M$ & 127 & 86 & 157 & 102 & 147 & 93 & & \\
\hline & SCC & T/S & 151 & 89 & 171 & 100 & 138 & 87 & & 9,1 \\
\hline & SPD & $\begin{array}{l}\text { Sorgo } \\
\text { Soja }\end{array}$ & $\begin{array}{l}120 \\
124\end{array}$ & $\begin{array}{l}101 \\
105\end{array}$ & $\begin{array}{l}118 \\
128\end{array}$ & $\begin{array}{l}103 \\
102\end{array}$ & $\begin{array}{l}111 \\
118\end{array}$ & $\begin{array}{r}86 \\
105\end{array}$ & & \\
\hline \multirow[t]{3}{*}{ LVd } & & Sorgo & 94 & 76 & 100 & 80 & 101 & 77 & \multirow[b]{2}{*}{11,3} & \multirow[b]{2}{*}{5,6} \\
\hline & SCC & Soja & 93 & 80 & 104 & 83 & & 85 & & \\
\hline & SPD & $\begin{array}{l}A / M \\
A+V / M+C\end{array}$ & $\begin{array}{l}74 \\
68\end{array}$ & $\begin{array}{l}71 \\
71\end{array}$ & $\begin{array}{l}62 \\
59\end{array}$ & $\begin{array}{l}61 \\
55\end{array}$ & $\begin{array}{l}53 \\
45\end{array}$ & $\begin{array}{l}54 \\
45\end{array}$ & & \\
\hline \multirow[t]{2}{*}{ PVd 1} & & $\mathrm{~A} / \mathrm{M}$ & 55 & 48 & 55 & 50 & 55 & 49 & \multirow{3}{*}{8,0} & \multirow{3}{*}{7,2} \\
\hline & $\mathrm{SCC}$ & $A+V / M+C$ & 50 & $4 /$ & 35 & 49 & 49 & 44 & & \\
\hline \multirow[t]{2}{*}{$P \vee d 2$} & \multirow[t]{2}{*}{ SPD } & $\begin{array}{l}A / M \\
A+V / M+C \\
G+M\end{array}$ & $\begin{array}{l}81 \\
67 \\
81\end{array}$ & $\begin{array}{l}77 \\
64 \\
79\end{array}$ & $\begin{array}{l}67 \\
65 \\
73\end{array}$ & $\begin{array}{l}65 \\
65 \\
69\end{array}$ & $\begin{array}{l}46 \\
61 \\
62\end{array}$ & $\begin{array}{l}52 \\
59 \\
61\end{array}$ & & \\
\hline & & & & & & & & & 15,4 & 11,4 \\
\hline
\end{tabular}

Quadro 5. F ator quanti dade de fósforo e contri buição de cada extrator à variação nesse fator em diferentes tipos de solo

\begin{tabular}{|c|c|c|c|c|c|c|c|}
\hline Solo(1) & Unidade & F ator quantidade ${ }^{(3)}$ & $\begin{array}{c}\Delta \mathrm{NaHCO}_{3}{ }^{(4)} \\
0,5 \mathrm{~mol} \mathrm{~L}^{-1}\end{array}$ & $\begin{array}{c}\Delta \mathrm{HCl} \\
1,0 \mathrm{~mol} \mathrm{~L}^{-1}\end{array}$ & $\begin{array}{c}\Delta \mathrm{NaOH} \\
0,1 \mathrm{~mol} \mathrm{~L}^{-1}\end{array}$ & $\begin{array}{c}\Delta \mathrm{NaOH} \\
0,5 \mathrm{~mol} \mathrm{~L}^{-1}\end{array}$ & $\sum \Delta^{(5)}$ \\
\hline \multirow[t]{2}{*}{ LVdf } & $\mathrm{mg} \mathrm{dm}^{-3}$ & 97 & 21 & -6 & 39 & 52 & 106 \\
\hline & $\%^{(2)}$ & & 20 & -6 & 36 & 49 & \\
\hline \multirow[t]{2}{*}{ LVd } & $\mathrm{mg} \mathrm{dm}-3$ & 122 & 17 & 7 & 117 & 19 & 160 \\
\hline & $\%$ & & 10 & 5 & 72 & 13 & \\
\hline \multirow[t]{2}{*}{$P \vee d 1$} & $\mathrm{mg} \mathrm{dm}^{-3}$ & 54 & 18 & 2 & 61 & 3 & 83 \\
\hline & $\%$ & & 22 & 2 & 73 & 3 & \\
\hline \multirow[t]{2}{*}{$P \vee d 2$} & $\mathrm{mg} \mathrm{dm}^{-3}$ & 51 & 17 & 3 & 45 & 1 & 66 \\
\hline & $\%$ & & 26 & 5 & 68 & 1 & \\
\hline
\end{tabular}


No SPD, a sucessão trigo/soja, por ter sido adubada duas vezes ao ano, apresentou quantidade de fósforo total adicionada elevada, $792 \mathrm{~kg} \mathrm{ha}-1$. Destes, $374 \mathrm{~kg} \mathrm{ha-1}$ foram exportados pelos grãos, representando $47 \%$ dototal; $40 \mathrm{~kg}$ ha-1 foram perdi dos por erosão (5\%) e $213 \mathrm{~kg} \mathrm{ha-1}$ permaneceram no solo emformas potencialmentedisponíveis, representando $27 \%$ do fósforo aplicado. Assim, apenas $21 \%$ daquele fósforo aplicado passou para formas de fósforo nãolábil, provavel mente como fosfato de cál cio de baixa sol ubilidade (Magid, 1993; Rheinheimer et al., 1999). Na sucessão aveia/milho, adicionaram-se 612 kg ha-1 de fósforo, tendo sido $276 \mathrm{~kg} \mathrm{ha-1}$ (45\%) exportados pel os grãos, $40 \mathrm{~kg}$ ha-1 (6\%) saído do sol o por erosão e230 kg ha-1 (38\%) estão em formas potencialmente disponíveis. Nessa sucessão, somente $11 \%$ do fósforo total adicionado não foi recuperado durante os dezoito anos de cultivo. A rotação de culturas, por ter menos cultivos adubados, recebeu $552 \mathrm{~kg}$ ha-1 de fósforo. Como as produtividades médias das gramíneas (trigo e milho) foram maiores do que nas sucessões, a quantidade exportada subiu para
$316 \mathrm{~kg} \mathrm{ha}-1$ (57\%). A quantidade de fósforo que permaneceu no solo em formas potencialmente disponíveis foi de $170 \mathrm{~kg} \mathrm{ha}^{-1}$ (31\%), que, juntamente com os 40 kg ha-1 (7\%) defósforo perdido por erosão, resultou numa recuperação de $95 \%$ do fósforo adicionado. A recuper ação do fósforo adicionado foi a mesma para o SCC, atingindo 79 e $89 \%$ nas sucessões trigo/soja e aveia/milho, respectivamente (Quadro 6).

Esses dados indicam que, em solos que foram adubados por vários anos, como a maioria dos solos hojecultivados noSPD, o fósforo que não éexportado pelas colheitas permanece no solo em formas potencialmente disponíveis, uma vez que as perdas por erosão são pequenas e os sítios de adsorção de fosfatos com maior energia já estão saturados, como também observaram Guo \& Yost (1998). Adicional mente, sistemas de cultivos que envolvem culturas que não exportam grãos e com maior habilidade de reciclagem de fósforo, como, por exemplo, a aveia, podem aumentar a percentagem de recuperação dos fertilizantes fosfatados apl icados.

Quadro 6. Balanço do fósforo no Latossolo Vermelho distroférrico típico nos sistemas de cultivo convencional e plantio direto cultivado com as sucessões trigo/soja, aveia/milho e rotação de cultura

\begin{tabular}{|c|c|c|c|c|c|c|c|c|c|c|c|c|c|}
\hline \multirow{3}{*}{$\begin{array}{l}\text { Preparo } \\
\text { sucessão }\end{array}$} & \multirow{3}{*}{ Cultura } & \multirow{3}{*}{$\begin{array}{c}\text { Número } \\
\text { de } \\
\text { cultivos }\end{array}$} & \multicolumn{2}{|c|}{ P adicionado } & \multirow{2}{*}{$\begin{array}{l}\text { Produtividade } \\
\text { média }\end{array}$} & \multirow{2}{*}{$\begin{array}{c}\text { Teor de } P \\
\text { no grão }\end{array}$} & \multirow{2}{*}{$\begin{array}{c}\mathbf{P} \\
\text { exportado }\end{array}$} & \multirow{2}{*}{$\begin{array}{l}\text { Perda por } \\
\text { erosão(3) }\end{array}$} & \multirow{2}{*}{$\begin{array}{c}\mathbf{P}^{(4)} \\
\text { disponível }\end{array}$} & \multicolumn{4}{|c|}{$\mathbf{P}^{(5)}$} \\
\hline & & & Cultivo & Total & & & & & & \multicolumn{2}{|c|}{ Recuperado } & \multicolumn{2}{|c|}{ Não recuperado } \\
\hline & & & & $-\mathrm{kgh}$ & $a^{-1}$ & $\mathrm{~g} \mathrm{~kg}^{-1}$ & & $-\mathrm{kg} \mathrm{ha}^{-1}$ & - & $\mathrm{kg} \mathrm{ha}^{-1}$ & $\%$ & $\mathrm{~kg} \mathrm{ha}^{-1}$ & $\%$ \\
\hline \multirow{3}{*}{$\mathrm{T} / \mathrm{S}^{(2)}$} & Trigo & 18 & 22 & 396 & 1.380 & 6,0 & 149 & & & & & & \\
\hline & Soja & 18 & 22 & 396 & 2.350 & 5,0 & 211 & & & & & & \\
\hline & Somatório & & & 792 & & & 360 & 89 & 177 & 626 & 79 & 166 & 21 \\
\hline \multirow{4}{*}{$A / M$} & Trigo & 8 & 22 & 176 & 1.380 & 6,0 & 66 & & & & & & \\
\hline & Soja & 8 & 22 & 176 & 2.350 & 5,0 & 94 & & & & & & \\
\hline & Milho & 10 & 26 & 260 & 3.170 & 3,0 & 95 & & & & & & \\
\hline & Somatório & & & 612 & & & 255 & 89 & 202 & 546 & 89 & 66 & 11 \\
\hline \multicolumn{14}{|l|}{ SPD } \\
\hline \multirow{3}{*}{$\mathrm{T} / \mathrm{S}$} & Trigo & 18 & 22 & 396 & 1.300 & 6,0 & 140 & & & & & & \\
\hline & Soja & 18 & 22 & 396 & 2.600 & 5,0 & 234 & & & & & & \\
\hline & Somatório & & & 792 & & & 374 & 40 & 213 & 627 & 79 & 165 & 21 \\
\hline \multirow{4}{*}{$A / M$} & Trigo & 8 & 22 & 176 & 1.300 & 6,0 & 62 & & & & & & \\
\hline & Soja & 8 & 22 & 176 & 2.600 & 5,0 & 104 & & & & & & \\
\hline & Milho & 10 & 26 & 260 & 3.670 & 3,0 & 110 & & & & & & \\
\hline & Somatório & & & 612 & & & 276 & 40 & 230 & 546 & 89 & 66 & 11 \\
\hline \multirow{4}{*}{ Rotação } & Trigo & 6 & 22 & 132 & 1.750 & 6,0 & 63 & & & & & & \\
\hline & Soja & 12 & 22 & 264 & 2.600 & 5,0 & 156 & & & & & & \\
\hline & Milho & 6 & 26 & 156 & 5.370 & 3,0 & 97 & & & & & & \\
\hline & Somatório & & & 552 & & & 316 & 40 & 170 & 526 & 95 & 26 & 5 \\
\hline
\end{tabular}

(1) SCC = sistema cultivo convencional; SPD = sistema plantio direto. ${ }^{(2)} \mathrm{T} / \mathrm{S}=$ trigo/soja desde 1979; A/M =trigo/soja até a safra 85/86 e depois aveia/milho; Rotação = soja e milho no verão etrigo, aveia, tremoço, colza eaveia +trevo no inverno. (3) Considerando perda de 1 e $3 \mathrm{t} \mathrm{ha}^{-1} \mathrm{ano}^{-1}$ de solo e taxa de enriquecimento de 2 e $1,5 \mathrm{mg} \mathrm{dm}^{-3}$ de P no sedimento por mg $\mathrm{dm}^{-3}$ de P no solo para o SPD e SCC, respectivamente (Sengranfredo, 1995). ${ }^{(4)} \mathrm{P}$ disponível = extração sucessiva com resina em membrana, ponderando-se os teores e as camadas amostradas. ${ }^{(5)} \mathrm{P}$ não recuperado $=\mathrm{P}$ adicionado $-(\mathrm{P}$ exportado $+\mathrm{P}$ erodido $+\mathrm{P}$ recuperado). 


\section{CONCLUSÕES}

1. A fração de fósforo inorgânico extraída com $\mathrm{HCl}$ $1,0 \mathrm{~mol} \mathrm{L-1,} \mathrm{num} \mathrm{esquema} \mathrm{seqüencial,} \mathrm{foi} \mathrm{indispo-}$ nível.

2. As frações de fósforo inorgânico extraídas pelo $\mathrm{NaHCO}_{3} 0,5$ e NaOH 0,1 mol L-1 foram fontes potenciais de fósforo biodisponível, independentemente do tipo desolo, método depreparo esucessão decultura, enquanto o extraído pelo $\mathrm{NaOH}$ 0,5 mol L-1 também o foi para os Latossolos com altos teores de argila e óxidos de ferro.

3. O fator quantidade de fósforo no sol o pode ser estimado pela extração com $\mathrm{NaOH} 0,1 \mathrm{~mol} \mathrm{L-1}$.

4. Foram recuperados 79 , 89 e $95 \%$ do fósforo adicionado no Latossolo Vermel ho distroférricotípico na sucessão trigo/soja, aveia/milho e rotação de culturas, respectivamente.

\section{AGRADECIMENTOS}

Ao professor J oão Mielniczuk (UFRGS) e aos pesquisadores Rainoldo Alberto Kochhann (E mbrapa - Centro Nacional de Pesquisa de Trigo), Armando Dalla Rosa eJ oãoBecker (COTRISA), pela permissão de uso das áreas experimentais as quais serviram de base para este trabalho.

\section{LITERATURA CITADA}

AGBENIN, J .O. \& TIESSEN, H. Phosphorus transformations in a toposequence of Lithosols and Cambisols from semi-arid northeastern Brazil. Geoderma, 62:345-362, 1994.

AMADO, T.; MIELNICZUK, J .; FERNANDES, S.B. \& BAYER, C. Culturas de cobertura, acúmulo de nitrogênio total no solo eprodutividadedemilho. R. Bras. Ci. Solo, 23:679-686, 1999.

ARAÚJ O, M.S.B. \& SALCEDO, I.H. Formas preferenciais de acumulação de fósforo em solos cultivados com cana-deaçúcar na região do Nordeste. R. Bras. Ci. Solo, 21:643650, 1997.

BALL-COELHO, B.; SALCEDO, I.H.; TIESSEN, H . \& STEWART, J.W.B. Short and long-term phosphorus dynamics in a fertilized Ultisol under sugarcane. Soil Sci. Soc. Am. J., 57:1027-1034, 1993.

BAYER, C. Dinâmica da matéria orgânica em sistemas demanejo de solos. PortoAlegre, Universidade Federal do Rio Grande do Sul, 1996. 240 p. (Tese de Doutorado)

BECK, M.A. \& SANCHEZ, P.A. Soil phosphorus fraction dynamics during 18 years of cultivation on a Typic Paleudult. Soil Sci. Soc. Am. J ., 58:1424-1431, 1994.

CAMPELLO, M.R.; NOVAIS, R.F.; FERNANDEZ, I.E.; FONTES, M.P.F. \& BARROS, N.F. Avaliação da reversibilidade de fósforo não-lábil para lábil em solos com diferentes características. R. Bra. Ci. Solo, 18:157-165, 1994.
CHANG, S.C. \& J ACKSON, M.L. Fractionation of soil phosphorus. Soil Sci., 84:133-144, 1957.

CONDRON, L.M.; GOH, K.M. \& NEWMAN, R.H. Nature and distribution of soil phosphorus as revealed by a sequential extraction method followed by 31P nuclear magnetic resonance analysis. J. Soil Sci., 36:199-207, 1985.

CROSS, A.F. \& SCHLESINGER, W.H. A literature review and evaluation of the Hedley fractionation: Applications to the biogeochemical cycle of soil phosphorus in natural ecosystems. Geoderma, 64:197-214, 1995.

DALLA ROSA, A. Práticas mecânicas e culturais na recuperação de características físicas de solos degradados pel o cultivo soloSantoÂngelo(LDR). PortoAlegre, UniversidadeFederal do Rio Grande do Sul, 1981. 136p. (Tese de Mestrado)

DALLA ROSA, A.; MERTEN, G.H. \& MIELNICZUK, J. Produtividade do trigo e da soja em sistemas de culturas e preparos de solo. Trigo Soja, 113:14-18, 1991.

DEAN, L.A. Distribution of the forms of soil phosphorus. Soil Sci. Soc. Am. Proc., 2:223-227, 1937.

DENARDIN, E. \& KOCHHANN, R.A. Efeito de diferentes sistemas de preparo do solo no rendimento da cevada e na população de insetos subterrâneos. Passo Fundo, Empresa Brasileira de Pesquisa Agropecuária, 1998. 28p. ( Relatório Técnico Anual)

DICK, W.A. \& TABATABAI, M.A. Determination of orthophosphate in aqueous solutions containing labile organic and inorganic phosphorus compounds. J. Environ. Qual., 6:82-85, 1977.

GUO, F.\& YOST, R.S. Partitioning soil phosphorus into three discrete pools of differing availability. Soil Sci., 163:822833, 1998.

HAGGAR, J.P.; WARREN, G.P.; BEER, J.W. \& KASS, D. Phosphorus availability under alley cropping and mulched and unmulched sole cropping systems in Costa Rica. Plant Soil, 137:275-283, 1991.

HEDLEY, M.J .; STEWART, J.W.B. \& CHAUHAN, B.S. Changes in inorganic and organic soil phosphorus fractions induced by cultivation practices and by laboratory incubations. Soil Sci. Soc. Am. J ., 46:970-976, 1982.

KROTH, P.L. Disponibilidade de fósforo no solo para plantas e fatores que afetam a extração por resina de troca em membrana. Porto Alegre, Universidade Federal do Rio Grande do Sul, 1998. 168 p. (Tese de Mestrado)

MAGIG, J . Vegetation effects on phosphorus fraction in set-aside soils. Plant Soil, 149:111-119, 1993.

MCKEAN, S.J . \& WARREN, G.P. Determination of phosphate desorption characteristics in soils using successsive resin extractions. Comm. Soil Sci. Plant Anal., 27:2397-2417, 1996.

MERTEN, G.H. Rendimento de grãos e distribuição do sistema radicular das culturas sob diferentes sistemas de manejo em um Oxissolo (LDR). Porto Alegre, Universidade Federal do Rio Grande do Sul, 1988. 178p. (Tese de Mestrado)

MURPHY, J \& \& RILEY, J .P. A modified single solution method for determination of phosphate in natural waters. Anal. Chem. Acta, 27:31-36, 1962. 
RHEINHEIMER, D.S. \& ANGHINONI,I. Distribuição do fósforo inorgânico em sistemas de manejo de solo. Pesq. Agroc. Bras. (enviado para publicação, 1999)

SANYAL, S.K. \& DATTA, S.K. Chemistry phosphorus transformation in soil. Adv. Soil Sci., 16:1-120, 1991.

SCHMIDT, J .P.; BUOL, S.W. \& KAMPRATH, E.J . Soil phosphorus dynamics during seventeen years of continuous cultivation: fractionation analyses. Soil Sci. Soc. Am. J ., 60:1168-1172, 1996.

SELLES, F.; KOCHHANN, R.A.; DENARDIN, J .E.; ZENTNER, R.P. \& FAGANELLO, A. Distribution of phosphorus fractions in Brazilian Oxisol under different tillage systems. Soil Till. Res., 44:23-34, 1997.

SENGANFREDO, M.L. Sistemas de culturas adaptadas à produtividade do milho e conservação do solo. Santa Maria, Universidade Federal de Santa Maria, 1995. 100p. (Tese de Mestrado)
SILVA, F.C. \& RAIJ , B.van. Disponibilidade de fósforo em solos avaliada por diferentes extratores. Pesq. Agropec. Bras., 34:267-288, 1999.

SMILEY, R.W. Rhizosphere $\mathrm{pH}$ as influenced by plants, soils, and nitrogen fertilizers. Soil Sci. Soc. Am. Proc., 38:795801, 1974.

TESTA, V.M. Características químicas de um solo Argissolo Vermel ho distrófico Típico, nutrição e rendimento de milho afetados por sistemas de culturas. Porto Alegre, Universidade Federal do Rio Grande do Sul, 1989. 146 p. (Tese de Mestrado)

TIESSEN, H.; STEWART, W.B. \& COLE, C.V. Pathways of phosphorus transformations in soils of differing pedogenesis. Soil Sci. Soc. Am. J ., 48:853-858, 1984.

ZHANG, T.Q. \& MacKENZIE, A.F. Changes of soil phosphorus fractions under long-term corn monoculture. Soil Sci. Soc. Am. J ., 61:485-493, 1997. 